\section{Fórum PrEP: um debate on-line sobre uso da profilaxia pré-exposição no Brasil}

\author{
PrEP Forum: an on-line debate on pre-exposure \\ prophylaxis in Brazil
}

Fórum PrEP: un debate on-line sobre uso de la
profilaxia pre-exposición en Brasil
Artur Acelino Francisco Luz Nunes Queiroz 1 Alvaro Francisco Lopes de Sousa 1

doi: 10.1590/0102-311X00112516

\section{Resumo}

Este estudo objetivou identificar conteúdos promotores de saúde voltados à prevenção do HIV/aids, em postagens veiculadas em um grupo do Facebook destinado a debates sobre uso da profilaxia pré-exposição (PrEP). Trata-se de um estudo observacional, prospectivo, desenvolvido por meio de observação sistemática não-participativa. Durante o período de julho de 2015 a junho de 2016, todas as postagens realizadas no grupo foram catalogadas e formaram um corpus. Tudo foi processado no software IRaMuTeQ e analisado pela classificação hierárquica descendente. Os dados obtidos foram agrupados em três classes: (1) prevenção do HIV/aids: discutindo sobre profilaxia, tratamento, público alvo e efeitos colaterais; (2) acesso universal a PrEP no Brasil: discutindo as responsabilidades governamentais; (3) compra on-line da truvada: expondo uma situação de vulnerabilidade. Os achados alertam para um problema de saúde pública em potencial, e fornecem subsídios para compreender aspectos facilitadores e barreiras ao uso da PrEP no país, por meio da identificação de conteúdo promotores de saúde articulados com marcadores individuais, sociais e institucionais.

Profilaxia Pré-exposição; Rede Social; HIV; Síndrome de Imunodeficiência Adquirida

\author{
Correspondência \\ A. A. F. L. N. Queiroz \\ Escola de Enfermagem de Ribeirão Preto, Universidade de \\ São Paulo. \\ Av. dos Bandeirantes 3900, Campus Universitário, \\ Ribeirão Preto, SP 14040-902, Brasil. \\ arturqueiroz@usp.br \\ 1 Escola de Enfermagem de Ribeirão Preto, Universidade de São \\ Paulo, Ribeirão Preto, Brasil.
}




\section{Introdução}

Entre as atuais estratégias de prevenção biomédica contra a infecção pelo HIV, a profilaxia pré-exposição (PrEP), vem se destacando pela eficácia apresentada nos ensaios clínicos desenvolvidos, com redução no risco de infecção que varia de $92 \%{ }^{1}$ a $100 \%$ 2, a depender da adesão à terapêutica ${ }^{3}$. Seu uso é particularmente recomendado para populações-chave vulneráveis a infecção, dentre os quais se destacam homens que fazem sexo com homens (HSH) 1.

O uso de PrEP por HSH em situação de risco já é uma realidade em países desenvolvidos, e conta com a anuência de agências governamentais norte-americanas 4, além de órgãos regulatórios de países como Reino Unido e França 5. A Organização Mundial da Saúde (OMS) também emitiu recomendações e diretrizes para uso da PrEP por populações mais vulneráveis de países em desenvolvimento, em combinação com outras formas de prevenção 6 .

A PrEP, comercializada nos Estados Unidos como "truvada", é uma combinação de dois medicamentos em um único comprimido: o fumarato de tenofovir desproxila (TDF - 300mg) e a emtricitabina (FTC - 200mg), e sua utilização é feita diariamente, por via oral 7. Embora atualmente somente essa combinação esteja aprovada para uso, estudos sobre a PrEP utilizando outros medicamentos e vias de administração vêm sendo desenvolvidos 3 .

No Brasil, o uso e a comercialização do medicamento não estão legalizados, embora nos últimos anos o debate e as ações em relação a sua implantação tenham tido alguns desdobramentos importantes. Em 2015, o Ministério da Saúde anunciou o desenvolvimento do primeiro estudo nacional com HSH visando à distribuição de PrEP gratuitamente pelo Sistema Único de Saúde (SUS) em hospitais e em postos especializados no tratamento e prevenção de infecções sexualmente transmissíveis (IST)/ aids, diferente do que ocorre em outros países onde o medicamento é comercializado ${ }^{8}$. No entanto, já há relatos do seu uso por meio da compra de outros países ${ }^{9}$, sem que haja prescrição ou acompanhamento médico, o que pode ocasionar danos aos seus usuários, ou ainda ter sua eficácia diminuída pelo uso inadequado.

Em julho de 2016, durante a 21ạ Conferência Internacional de Aids, realizada em Durban, na África do Sul, a diretoria do Departamento de DST, Aids e Hepatites Virais anunciou que o Brasil, por meio do Ministério da Saúde, ofereceria até o fim de 2016 o medicamento pelo SUS. No entanto, a Comissão Nacional de Incorporação de Tecnologias do SUS (Conitec) decidiu aguardar a aprovação da droga como método de prevenção junto à Agência Nacional de Vigilância Sanitária (Anvisa) 10.

A incorporação não foi viabilizada, porque o medicamento precisa ser registrado como método de prevenção, e não somente tratamento, o que requer mudanças em sua bula. Essa situação ocasionou uma nova previsão de implantação da PrEP pelo SUS (fevereiro de 2017) 11, que tem gerado desconforto naqueles que aguardavam a implantação do medicamento, seja os que pretendiam iniciar seu uso, seja aqueles usuários que tiveram o uso interrompido pela finalização dos estudos realizados no país.

Toda essa problemática revela a necessidade de implementação de estratégias direcionadas a essa população, em sua atual situação de saúde. Nesse contexto, o uso das tecnologias de informação e comunicação tem permitido a prestação de cuidados de forma remota, principalmente no que concerne à educação em saúde em populações de difícil acesso, como HSH ${ }^{9}$. Tais tecnologias podem contribuir para suprimir vulnerabilidades e permitir o acompanhamento desses sujeitos.

Este estudo objetivou identificar conteúdos promotores de saúde voltados à prevenção do HIV/ aids, em postagens veiculadas em um grupo do Facebook destinado a debates sobre o uso da PrEP. Para isso, foi adotado como conteúdo promotor de saúde "a comunicação de informações capazes de serem transformadas em recursos para tomada de decisão em favor da melhoria da qualidade de vida e da saúde de indivíduos ou grupos" 12 (p. 458).

\section{Metodologia}

Trata-se de um estudo observacional, prospectivo, desenvolvido por meio de observação sistemática não participativa em um grupo do Facebook destinado a "conversar e aprender mais sobre PrEP (profilaxia pré-exposição), o método de prevenir o HIV, tomando um comprimido todo dia”. 
O grupo está vinculado a essa rede social (https://www.facebook.com/groups/forumprep/) desde 2015, e possuía 305 usuários, sendo a maioria (93\%) do sexo masculino. O grupo é fechado, exigindo aprovação do moderador para se tornar participante, e seu propósito é discutir aspectos relacionados ao HIV/aids, com ênfase na PrEP. Para a pesquisa, solicitou-se autorização dos moderadores do grupo, que concordaram com sua realização. Apesar de fazer uma triagem das investigações relacionadas a PrEP vigentes no Brasil, oficialmente o grupo não é vinculado a nenhuma iniciativa de pesquisa.

Assim, durante o período de um ano (julho de 2015 a junho de 2016), todas as postagens realizadas no grupo, bem como as respostas, foram catalogadas e formaram um corpus, tendo três variáveis fixas: categoria (postagem ou resposta), mês de postagem e idioma.

Os dados agrupados tiveram tratamento estatístico pelo software IRaMuTeQ (acrônimo de Interface de $R$ pour les Analyses Multidimensionnelles de Texteset de Questionnaires; http://www.iramuteq. org/), o qual recorre a análises lexicais sofisticadas para expor os resultados 13,14,15. Foi formado um dicionário que permitisse o agrupamento de vocábulos por similaridade semântica existente entre as palavras, o que permitiu melhorar o aproveitamento e condensar determinados vocábulos.

Os dados foram tratados utilizando-se o método da classificação hierárquica descendente 13,14,16, segundo o qual os textos são classificados em função de seus respectivos vocabulários, e o conjunto deles se divide pela frequência das formas reduzidas. A partir de matrizes que cruzaram segmentos de textos e palavras (repetidos testes qui-quadrado), aplicou-se o método da classificação hierárquica descendente, e foi obtida uma classificação estável e definitiva. Essa classificação permitiu a obtenção de classes de segmentos de texto com vocabulário semelhante entre si, mas, ao mesmo tempo, diferente dos segmentos de texto das outras classes.

O estudo obedeceu a todos os preceitos éticos nacionais e internacionais que regem a pesquisa, não havendo identificação dos participantes do grupo, ou de qualquer característica que permitissem a sua identificação (parecer 1.523.003). Baseado na Resolução $n^{\circ}$ 510/2016 17, que trata das especificidades éticas das pesquisas nas ciências humanas e sociais, solicitou-se dispensa do Termo de Consentimento Livre e Esclarecido por tratar-se de pesquisa que utiliza informações de acesso público.

\section{Resultados}

Os dados obtidos deram origem a um conjunto de 39.085 palavras, tendo o corpus final uma extensão de 81 páginas. O software reconheceu a separação do corpus em 1.173 unidades de contexto elementar, a partir de 531 unidades de contexto inicial, composta por postagens ou respostas. Foram registradas 36.215 ocorrências com aproveitamento de $86,7 \%$ do corpus total.

Por meio da classificação hierárquica descendente, identificaram-se e analisaram-se os domínios textuais, procedendo à interpretação dos significados, dando-lhes nomes a partir dos seus respectivos sentidos em classes (Figura 1).

\section{Classe 1 - prevenção do HIV/aids: discutindo sobre profilaxia, tratamento, público alvo e efeitos colaterais}

A presente classe comporta a maioria dos vocábulos que fizeram parte do corpus inicial. Acreditamos que essa concentração elevada $(73,2 \%)$ se dá por ser uma classe conceitual, que reúne os significados atribuídos a diversas palavras como PrEP, PEP (profilaxia pós-exposição), eficácia, população-chave, entre outros. Além disso, o entendimento do conceito propicia a discussão de outros fatores associados como orientação sexual, efeitos colaterais, acesso universal ao tratamento e profilaxia.

A discussão, baseada em conceitos e seu entendimento, acontecia em diversos idiomas e indicava sujeitos (membros do grupo) com alto potencial de empoderamento. Estes eram capazes não somente de buscar sua própria saúde, mas de avaliar criticamente tal situação e disseminar conteúdo a outros em semelhante estado de vulnerabilidade.

Conteúdos relacionados à necessidade de "avaliar a adesão" ou à prioridade do acesso grátis pelo SUS para populações prioritárias, como homens que fazem sexo com homens, são recorrentes. 
Figura 1

Estrutura temática dos conteúdos promotores de saúde voltados ao debate da profilaxia pré-exposição (PrEP) veiculados em postagens de um grupo no Facebook.

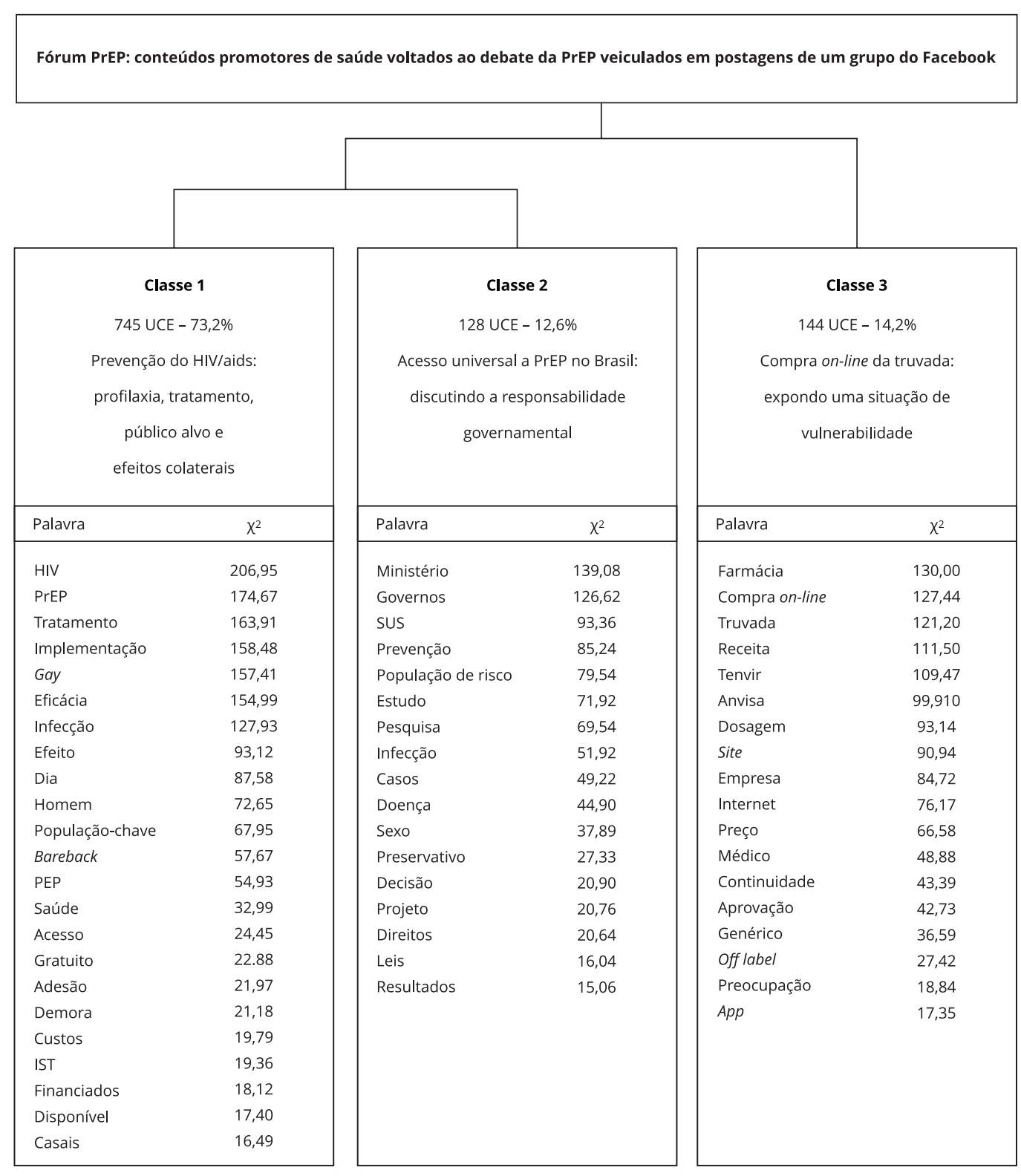

Anvisa: Agência Nacional de Vigilância Sanitária; IST: infecções sexualmente transmissíveis; PEP: profilaxia pós-exposição; SUS: Sistema Único de Saúde; UCE: unidade de contexto elementar.

\section{Classe 2 - acesso universal a PrEP no Brasil: discutindo as responsabilidades governamentais}

Essa classe comporta-se como complementar à anterior, pois, cientes dos benefícios da PrEP, da sua importância para a prevenção do HIV/aids, principalmente em populações-chave como HSH, os participantes buscam discutir e elencar responsabilidades. 
Para isso, utilizam a experiência de outros países que adotaram essa estratégia, como França e Reino Unido, e outros sistemas de saúde, por meio de notícias em jornais de grande circulação ou até mesmo textos científicos nos mais variados idiomas.

É comum a discussão sobre os estudos que estão sendo realizados no Brasil, para avaliar a adesão da PrEP. O avanço de outros agravos de transmissão sexual, como a sífilis e as hepatites, é um tópico recorrente. Os participantes também discutem estratégias de implementação e distribuição da PrEP, como fornecê-la em conjunto com o preservativo em consultas de rotina, que poderiam ser acompanhadas de testes rápidos para outras IST.

\section{Classe 3 - compra on-line da truvada: expondo uma situação de vulnerabilidade}

A terceira classe destaca-se do segmento formado pelas classes 1 e 2, englobando-as. Isso, pode ser explicado pelo fato de o seu conteúdo comportar uma alternativa aos usuários que sabem da eficácia da PrEP, mas que não participam de nenhum estudo nacional que a forneça.

Nessa situação, a alternativa é a compra da truvada em farmácias on-line do exterior por preços elevados. Grande parte dos conteúdos dessa classe dizem respeito a dosagem, composição, preço do medicamento e principalmente efeitos colaterais.

Por não haver um acompanhamento médico, surgem dúvidas e questionamentos dos usuários quanto ao uso, efeito e eficácia. Essas dúvidas são expostas, trabalhadas e discutidas por outros membros. Enxerga-se, claramente, uma falha programática, em que, ao se tentar fugir de uma situação de vulnerabilidade individual (comportamentos de riscos), os sujeitos, acidentalmente, passam a conviver com outra situação de vulnerabilidade: uso de um medicamento potente, sem o devido acompanhamento médico. Alguns participantes utilizam o medicamento há bastante tempo, enquanto se observam, cada vez mais, outros sujeitos iniciando o seu uso.

\section{Discussão}

O presente estudo é pioneiro ao apropriar-se desse objeto de estudo vinculado às redes sociais como campo de pesquisa, no cenário nacional. Dessa forma, esta pesquisa possui elevado potencial de inovação e apoio a estratégias de implementação, captação e aderência da PrEP no Brasil.

Nossos achados apontam para um problema de saúde pública em potencial e fornecem subsídios para compreender aspectos facilitadores e barreiras ao uso da PrEP no país, por meio da identificação de conteúdos promotores de saúde articulados com marcadores individuais, sociais e institucionais, imersos em um grupo reunido em uma rede social.

Os debates envolvendo a PrEP têm aumentado em todo o mundo, e cada vez mais extrapolam os espaços acadêmicos. Enquanto países desenvolvidos avançam nos debates, países em desenvolvimento, que vêm registrando uma maior proporção de casos novos para o HIV/aids, tal como o Brasil 18, ainda estudam estratégias de implantação e adesão.

Enquanto isso, a conscientização sobre a PrEP, seus benefícios e efeitos colaterais penetram cada vez mais os meios de comunicações em massa, tornando-se de conhecimento para a população em geral, utilizando-se principalmente do potencial da Internet. A rede mundial de computadores contribui significativamente, por proporcionar não somente a divulgação de conteúdos em escala mundial, mas também por permitir a reunião de diversos sujeitos com características comuns de forma on-line e prática, em um ambiente que propicia relações mais horizontais e permite diálogos democráticos 19 .

Nesse sentido, o uso de tecnologias emergentes de informação e comunicação em massa para melhorar ou permitir a saúde (e-Health) vêm destacando-se pelo alto potencial de inovação e propagação, no que concerne à promoção da saúde de forma remota, principalmente relacionada à educação em saúde em populações de difícil acesso 20 . Esta pesquisa comprovou tal potencial, ao mostrar diferentes sujeitos, de determinados países, reunindo-se para discutir sobre assunto pertinente a sua saúde sexual.

A consciência do potencial da PrEP a torna diferencial, principalmente para grupos-chave na epidemia do HIV ou ainda para aqueles que se envolvem em comportamentos de alto risco, como tra- 
balhadores do sexo e HSH que praticam sexo anal desprotegido 20,21. Isso faz com que os participantes busquem por conteúdos relacionados e que se reúnam para discuti-los.

Nesse sentido, tópicos acerca da distribuição e do acesso da PrEP pelo SUS são uma das principais e mais frequentes discussões no grupo. O principal argumento utilizado é que, no Brasil, o acesso ao tratamento do HIV/aids é gratuito e universal pelo SUS desde 1996. Considerando que fazem parte da população-chave, no Brasil, sujeitos que se encontram em maior situação de vulnerabilidade, é dever do governo distribuí-lo gratuitamente, de preferência combinada com outras estratégias de prevenção e controle 22 .

Discutir a prevenção combinada reforça uma preocupação em aumentar os títulos protetores, sendo percebida como uma "proteção adicional". De fato, a prevenção combinada é a melhor opção, tanto para prevenir o HIV, quanto outras IST 23. No entanto, são comuns tópicos sobre situações em que o uso do preservativo não pode ser assegurado (como profissionais do sexo), rompimento do preservativo, ou ainda o sexo bareback, prática comum em alguns grupos de HSH e chave para o controle da infecção nesse segmento 24 , sendo necessários mais estudos que elucidem a relação entre uso de PrEP e essa prática.

Embora os participantes deem maior ênfase ao uso de PrEP em conjunto com o preservativo como prevenção combinada, ela não se restringe a esses dois componentes. A realização de testes para o HIV, estratégias comportamentais, o uso da PEP, o tratamento como prevenção e a circuncisão também fazem parte das estratégias de prevenção combinada 25.

Destaca-se a importância dada à PrEP em relacionamentos sorodiscordantes para o HIV, o que pode diminuir a "carga de medo" em uma possível relação sexual em que o preservativo estoure, facilitando o estabelecimento de parcerias para pessoas vivendo com HIV/aids 26,27.

A sensação de proteção e "sexo seguro" ocasionada pelo uso da PrEP vem sendo associada a uma diminuição no uso do preservativo, mesmo entre HSH que não usam PrEP, mas que o parceiro utilize. Isso pode levar a um aumento de outras doenças sexualmente transmissíveis, embora estudos realizados em países desenvolvidos demonstrem o contrário 28. No Brasil, série histórica fornecida pelo Ministério da Saúde destaca o aumento dos casos de sífilis e HIV entre a população masculina, sugerindo uma diminuição do uso do preservativo como algo muito anterior à PrEP 29,30.

A situação de vulnerabilidade, principalmente programática, vivida pelos participantes é um dos principais achados desta pesquisa e alerta para um grave problema: o uso de medicamentos (truvada), sem que haja acompanhamento médico ou exames de rotina para avaliar a sua eficácia. Essa situação obriga os participantes a refletir e discutir sobre a implantação e distribuição da PrEP no Brasil.

Usuários atuais da truvada relatam o alto preço como impeditivo para a uma boa adesão, especialmente em populações mais carentes. Para isso, utilizam da sua própria experiência de compra por meio de farmácias on-line no exterior. Muitos ressaltam o medo sobre a veracidade da truvada comercializada, temendo que as farmácias possam estar vendendo produto falso para aumentar os lucros. Nesse sentido, reforçam a importância de órgãos regulatórios como a Anvisa. No que concerne a uma possível comercialização desse medicamento em farmácias brasileiras, os usuários mostramse receosos de que essa estratégia possa ser afetada pela dificuldade do brasileiro em expressar a sua sexualidade, situação semelhante à vivida pelo preservativo 31 .

Discussões sobre a eficácia da PrEP são sempre presentes, subsidiadas por estudos publicados em revistas de circulação internacional. Os participantes discutem a relação eficácia-adesão, dose diária e manutenção de hábitos saudáveis. Os efeitos colaterais estão quase sempre presentes e geram grandes discussões. Muitos sujeitos que irão iniciar PrEP relatam medo dos efeitos colaterais e, principalmente, que sejam visíveis. No entanto, observa-se pouca (cefaleia e tontura) ou nenhuma reclamação de efeito colateral.

Enquanto alguns participantes diziam sentir-se bem com doses diárias, outros relatavam medo de que tal dose fosse "agressiva” ou até mesmo desgastante. Assim, discutiam mudanças na dose, frequência de uso ou uso apenas em situações especiais. Isso abria caminho para discussões sobre adesão, custos, disponibilidade e até mesmo resistência.

As preocupações expostas pelos participantes do grupo podem auxiliar no delineamento de futuras pesquisas com usuários ou candidatos ao uso da PrEP, baseando-se nas características descritas neste estudo. A viabilidade de pesquisas on-line também deve ser explorada, avançando em abordagens e metodologias dinâmicas ou ainda em intervenções feitas em ambientes virtuais. 
Esta pesquisa possui limitações. A principal delas refere-se à relação "Internet e democratização do conhecimento" e suas implicações na exclusão social. A exemplo disso, no grupo em estudo, os textos utilizados nas discussões estavam quase sempre no idioma inglês, o que exigia dos participantes um bom domínio da língua.

Destacamos, ainda, que a pesquisa se limita a reportar os achados de um grupo específico contido em uma rede social. Além disso, o método de coleta de dados adotado não possibilita aos pesquisadores controlar e manipular as condições nas quais estão interessados, de modo que, ao inibir a influência do pesquisador sobre as discussões, preserva-se o seu "estado natural".

\section{Conclusão}

Identificamos conteúdos promotores de saúde relacionados à prevenção do HIV/aids no grupo estudado, centrados e articulados com importantes marcadores, de cunho individual (percepção de risco e empoderamento), social (vulnerabilidades, inclusão, identidade sexual e posição socioeconômica) e institucional (papel e responsabilidade governamental).

Os participantes iniciavam e fomentavam discussões subsidiadas em textos publicados em periódicos científicos internacionais ou em jornais de grande circulação que envolviam desde a apresentação e o entendimento de alguns conceitos à necessidade de distribuição gratuita da PrEP pelo SUS. Os desdobramentos relacionados à implantação da PrEP no Brasil vêm causando "estresse" nos participantes e têm ocupado o centro das discussões.

Mudanças relacionadas a distribuição e comercialização da PrEP nas farmácias brasileiras ainda abrem margem para uma discussão abrangente sobre o papel da saúde, como instituição, na implementação e na disponibilidade de novas tecnologias de prevenção, sendo necessários estudos posteriores que avaliem a viabilidade dessa estratégia, bem como a aceitabilidade pela comunidade.

O Facebook configura-se como um importante espaço de interação que proporciona e propicia a circulação de discursos e transmissão de informações. Tal potencial deve ser mais bem explorado pelo campo da saúde.

\section{Colaboradores}

A. A. F. L. N. Queiroz e A. F. L. Sousa contribuíram na concepção, análise e interpretação dos dados, redação do artigo e revisão crítica relevante do conteúdo intelectual, tendo aprovado a versão final a ser publicada.

\section{Agradecimentos}

Aos participantes da pesquisa, em especial aos administradores do grupo, Artur Zalewska e Henrique Contreiras, pelo apoio.

\section{Referências}

1. Grant RM, Lama JR, Anderson PL, McMahan V, Liu A, Vargas L, et al. Preexposure chemoprophylaxis for HIV prevention in men who have sex with men. N Engl J Med 2010; 363:2587-99.

2. Grant RM, Anderson PL, McMahan V, Liu A, Amico KR, Mehrotraet M, et al. Uptake of preexposure prophylaxis, sexual practices, and HIV incidence in men and transgender women who have sex with men: a cohort study. Lancet Infect Dis 2014; 14:820-9.

3. Moss JA, Srinivasan P, Smith TJ, Butkyavichene I, Lopez G, Brooks AA, et al. Pharmacokinetics and preliminary safety study of pod-intravaginal rings delivering antiretroviral combinations for HIV prophylaxis in a macaque model. Antimicrob Agents Chemother 2014; 58:5125-35. 
4. Office of the Commissioner. FDA approves first drug for reducing the risk of sexually acquired hiv infection. http://www.fda.gov/ NewsEvents/Newsroom/PressAnnounce ments/ucm312210.htm (acessado em 18/Mai/ 2015).

5. McCormack S, Fidler S, Fisher M; British HIV Association; British Association for Sexual Health and HIV. The British HIV Association/ British Association for Sexual Health and HIV Position Statement on pre-exposure prophylaxis in the UK. Int J STD AIDS 2012; 23:1-4.

6. World Health Organization. Consolidated guidelines on HIV prevention, diagnosis, treatment and care for key populations. Geneva: World Health Organization; 2014.

7. Van der Elst EM, Mbogua J, Operario D, Mutua G, Kuo C, Mugo P, et al. High acceptability of HIV pre-exposure prophylaxis but challenges in adherence and use: qualitative insights from a phase I trial of intermittent and daily PrEP in at-risk populations in Kenya. AIDS Behav 2013; 17:2162-72.

8. PrEP Brasil Profilaxia Pré Exposição. Estudo PrEP Brasil. http://prepbrasil.com.br/pesquisa -prep-brasil/ (acessado em 03/Jan/2017).

9. Alves N, Cambriconi F. Sites vendem medicamento que evita a Aids. Agora São Paulo 2010; 25 nov. http://www.agora.uol.com.br/saopau lo/ult10103u835850.shtml (acessado em 18/ Mai/2015).

10. Agência Aids. Aids 2016: informação, atenção, sorrisos e simpatia - um dia de trabalho no estande brasileiro na Conferência de Durban, na África do Sul. http://agenciaaids.com.br/ho $\mathrm{me} /$ noticias/volta_item/25214 (acessado em 03/Jan/2017).

11. Buscato M. Comissão adia parecer sobre incorporação ao SUS de terapia para prevenir a AIDS. http://epoca.globo.com/vida/noti cia/2016/10/comissao-adia-parecer-sobre-in corporacao-ao-sus-de-terapia-para-preveniraids.html (acessado em 03/Jan/2017).

12. Cadaxa AG, Sousa MF, Mendonça AVM. Conteúdos promotores de saúde em campanhas de Aids no facebook dos ministérios da saúde do Brasil e do Peru. Rev Panam Salud Pública 2015; 38:457-63. 13.

13. Sousa AFL, Queiroz AAFLN, Oliveira LB, Valle ARMC, Moura MEB. Social representations of community-acquired infection by primary care professionals. Acta Paul Enferm 2015; 28:454-9.

14. Sousa AFL, Moura MEB, Batista OMA, Queiroz AAFLN, Oliveira LB, Andrade D. Representações sociais da enfermagem sobre biossegurança: saúde ocupacional e o cuidar prevencionista. Rev Bras Enferm 2016; 69:864-71.

15. Ratinaud P, Marchand P. Application of the ALCESTE method to the large corpus and stabilised lexical worlds or "cablegate", using IRAMUTEQ. http://lexicometrica.univ-paris 3.fr/jadt/jadt2012/Communications/Ratin aud,\%20Pierre\%20et\%20al.\%20-\%20Applica tion\%20de\%20la\%20methode\%20Alceste.pdf (acessado em 03/Jan/2017).
16. Reinert M. Une methode de classification descendante hierarchique: application a l'analyse lexicale par contexte. Cah Anal Donnees 1983; 8:187-98.

17. Conselho Nacional de Saúde. Resolução no 510, de 7 de abril de 2016. Dispõe sobre as normas aplicáveis a pesquisas em Ciências Humanas e Sociais. Diário Oficial da União 2016; 24 mai.

18. Joint United Nations Programme on HIV/ AIDS. AIDS by the numbers 2015. http://www. unaids.org/en/resources/documents/2015/ AIDS_by_the_numbers_2015 (acessado em 03/Jan/2017).

19. Rosser BS, Wilkerson JM, Smolenski DJ, Oakes JM, Konstan J, Horvath KJ. The future of Internet-based HIV prevention: a report on key findings from the Men's INTernet (MINTS-I, II) Sex Studies. AIDS Behav 2011; 15:91-100.

20. Etienne CF. La eSalud: aprovechar la tecnología en el camino hacia la cobertura universal de salud. Rev Panam Salud Pública 2014; 35:317-9.

21. Chakrapani V, Newman PA, Shunmugam M, Mengle S, Varghese J, Nelson R, et al. Acceptability of HIV pre-exposure prophylaxis (PrEP) and implementation challenges among men who have sex with men in India: a qualitative investigation. AIDS Patient Care STDS 2015; 29:569-77.

22. Departamento de DST, Aids e Hepatites Virais, Secretaria de Vigilância em Saúde, Ministério da Saúde. Protocolo de assistência farmacêutica em DST/HIV/Aids: recomendações do Grupo de Trabalho de Assistência Farmacêutica. Brasília: Ministério da Saúde; 2010. (Série A. Normas e Manuais Técnicos).

23. Golub SA, Kowalczyk W, Weinberger CL, Parsons JT. Preexposure prophylaxis and predicted condom use among high-risk men who have sex with men. J Acquir Immune Defic Syndr 2010; 54:548-55.

24. Davis O. A special issue of Sexualities: bareback sex and queer theory across three national contexts (France, UK, USA). Sexualities 2015; 18:120-6.

25. Cáceres CF, Koechlin F, Goicochea P, Sow PS, O'Reilly K, Mayer KH, et al. The promises and challenges of pre-exposure prophylaxis as part of the emerging paradigm of combination HIV prevention. J Int AIDS Soc 2015; 18(4 Suppl 3):19949.

26. Ware NC, Wyatt MA, Haberer JE, Baeten JM, Kintu A, Psaros C, et al. What's love got to do with it? Explaining adherence to oral antiretroviral pre-exposure prophylaxis for HIV-serodiscordant couples. J Acquir Immune Defic Syndr 2012; 59:463-8.

27. World Health Organization. Guidance on oral pre-exposure prophylaxis (prep) for serodiscordant couples, men and transgender women who have sex with men at high risk of HIV: recommendations for use in the context of demonstration projects. Geneva: World Health Organization; 2012. 
28. Lafeuillade A. HIV PreP opens the door to STDs: the pros. Journal of Virus Erradication 2016; 2 Suppl 1:1-7.

29. Secretaria de Vigilância em Saúde, Ministério da Saúde. Sífilis 2016. Boletim Epidemiológico 2016; 47:1-32.

\section{Abstract}

This study aimed to identify health-promoting contents focused on HIV/AIDS prevention in messages posted in a Facebook group for debates on the use of pre-exposure prophylaxis (PrEP). This was a prospective observational study using systematic non-participant observation. From July 2015 to June 2016, all the posts in the group were catalogued and formed a corpus. Everything was processed in IRaMuTeQ and analyzed by descending hierarchical classification. The collected data were grouped in three classes: (1) HIV/AIDS prevention: discussing prophylaxis, treatment, target public, and side effects; (2) universal access to PrEP in Brazil: discussing government responsibilities; (3) on-line purchase of truvada: exposing a situation of vulnerability. The findings call attention to a potential public health problem and provide backing for understanding facilitators and barriers to the use of PrEP in Brazil through the identification of health-promoting content linked to individual, social, and institutional markers.

Pre-exposure Prophylaxis; Social Networking; HIV; Acquired Immunodeficiency Syndrome
30. Secretaria de Vigilância em Saúde, Ministério da Saúde. Boletim Epidemiológico HIV AIDS 2016; ano V, n. 1.

31. Porto MP. Lutando contra a AIDS entre meninas adolescentes: os efeitos da Campanha de Carnaval de 2003 do Ministério da Saúde do Brasil. Cad Saúde Pública 2005; 21:1234-43.

\section{Resumen}

Este estudio tuvo como objetivo identificar contenidos promotores de salud, dirigidos a la prevención do VIH/SIDA, en publicaciones a través de un grupo de Facebook, destinado a debates sobre el uso de la profilaxia pre-exposición (PrEP). Se trata de un estudio observacional, prospectivo, desarrollado mediante observación sistemática noparticipativa. Durante el período de julio de 2015 a junio de 2016, todas las publicaciones realizadas en el grupo se catalogaron y formaron un corpus. Todo fue procesado en el software IRaMuTeQ y analizado por la clasificación jerárquica descendiente. Los datos obtenidos se agruparon en tres clases: (1) prevención del VIH/SIDA: discutiendo sobre profilaxia, tratamiento, público objetivo y efectos colaterales; (2) acceso universal la PrEP en Brasil: discutiendo las responsabilidades gubernamentales; (3) compra on-line de la truvada: exponiendo una situación de vulnerabilidad. Los hallazgos alertan de un problema de salud pública en potencia, y proporcionan apoyos para comprender aspectos facilitadores y barreras al uso de la PrEP en el país, mediante la identificación de contenidos promotores de la salud, en consonancia con marcadores individuales, sociales e institucionales.

Profilaxis Pre-exposición; Red Social; VIH; Sindrome de Inmunodeficiencia Adquirida

Recebido em 28/Jun/2016

Versão final reapresentada em 03/Jan/2017

Aprovado em 01/Fev/2017 